\title{
Carbon Nanotubes for Active Refrigeration and Cooling in Micro and Mesoscale Systems
}

\author{
Shuang Tang*
}

\begin{abstract}
With the development of micro and mesoscale electronics, photonics, and optoelectronics, heat management on such small scales is becoming important. In several emergent areas such as quantum computing and communication, satellite controlling, and sensing, the overall refrigeration or cooling rate is having a priority over energy consumption efficiency. Beyond the passive heat draining, active solid-state refrigeration and cooling based on the Peltier effect have been attractive. We are here to suggest the materials system of carbon nanotubes. By incorporating the energy sensitivity of transport into the thermopower investigation, we have found that the active refrigeration and cooling rates are better than or at least similar to the thermal conductivity of commonly used functional metals, especially at and above room temperature. The performance is also robust against the fluctuation of the band gap introduced during the batch synthesis of carbon nanotubes. Furthermore, the $p$ - and $n$-type regions have similar performance in thermoelectric refrigeration and cooling, which is providing convenience for the ultimate device design and manufacturing.
\end{abstract}

Keywords: Active Refrigeration and Cooling; Micro and Mesoscale; Carbon Nanotubes; Thermoelectrics; Peltier Effect. Received: 01 September 2021; Revised: 12 November 2021; Accepted: 17 November 2021.

Article type: Research article.

\section{Introduction}

The development of molecular science and nanotechnology has made it feasible to realize electronics ${ }^{[1,2]}$ and optoelectronics $^{[3-5]}$ based on micro and mesoscale materials. The refrigeration, ${ }^{[6,7]}$ cooling $^{[8,9]}$ and heat management ${ }^{[10,11]}$ for such small-scale systems are becoming an urgent issue. Besides passive thermal conduction, ${ }^{[12-14]}$ the solid-state active refrigeration and cooling technology based on the Peltier ${ }^{[15-18]}$ or the Nernst ${ }^{[19-21]}$ effects have been attractive. Traditionally, the figure of merit $\left(Z T=T \sigma S^{2} / \kappa\right)^{[22-24]}$ is used to characterize the energy consumption efficiency of such thermoelectric devices, where $\sigma$ is the electrical conductivity, $T$ is the temperature, $S$ is the thermopower, and $\kappa$ is the thermal conductivity.

Carbon nanotubes are promising for building micro and mesoscale heat management systems, due to their high achievable thermal conductivity ${ }^{[25-27]}$ and thermopower ${ }^{[28,29]}$ The carbon nanotubes have typical diameters ${ }^{[30,31]}$ that are small enough to be embedded with most nanoelectronic components, and lengths ${ }^{[32-34]}$ that are large enough for heat conduction over wide temperature gradient. Carbon nanotubes

College of Engineering, State University of New York Polytechnic

Institute, Albany, NY, 12203, USA.

*E-mail: tangs1@sunypoly.edu (S. Tang) with different chiralities may fall into metallic, ${ }^{[35,36]}$ semimetallic $^{[37,38]}$ or semiconducting ${ }^{[39-41]}$ phases. Furthermore, their noticeable mechanical strength and flexibility ${ }^{[2-44]}$ are also promising for building robust facilities.

Unfortunately, the potential of carbon nanotubes for micro and mesoscale refrigeration and cooling is not been fully explored. With the development of high- $k$ dielectric materials ${ }^{[45-47]}$ and the technology of carrier injection, ${ }^{[48-50]}$ the tuning of Fermi level and carrier concentration of carbon nanotubes with nanoscale diameters can be realized to traditional unreachable ranges, which may promise higher power density and active rate of refrigeration and cooling. As pointed out by Zebarjadi et al., ${ }^{[51]}$ in many important application environments including satellites and space stations, ${ }^{[52-56]}$ as well as quantum computing and quantum communications, ${ }^{[57-60]}$ the actual rate for refrigeration and cooling is of a much higher priority compared to the efficiency and the cost. However, traditional research has only focused on enhancing the figure of merit, without adequately emphasizing the active refrigeration and cooling rate. ${ }^{[51,61]}$ It is also challenging to study the thermoelectric parameters of single carbon nanotubes at different Fermi levels. Some researchers have used Mott's relation to obtain the thermopower, ${ }^{[62,63]}$ and some are estimating the electrical 
conductivity and thermopower by using a constant relaxation time approximation or by only considering the acoustic phonon scattering, ${ }^{[64-66]}$ despite the significant deviation from the real values. A more accurate method has been developed recently by Tang et al. ${ }^{[67,68]}$ by using the energy sensitivity of carrier transport and transport rate coefficient extracted from the experimental measurement.

In this paper, we will mainly study the potential of single-wall carbon nanotubes for micro and mesoscale refrigeration and cooling. The highest achievable active refrigeration and the cooling rate at both the room temperature of $300 \mathrm{~K}$ and the cryogenic temperature of $150 \mathrm{~K}$ are calculated for carbon nanotubes with chirality of $(5,5),(6,3)$ and $(8,0)$, which are metallic, ${ }^{[35,36]}$ semi-metallic ${ }^{[37,38]}$ and semiconducting, ${ }^{[39-41]}$ respectively.

\section{Method}

The widely adopted Mott's relation ${ }^{[62,63,67]}$ is useful in estimating the thermopower in a deep-band conducting regime, but cannot help us obtain the value of $S_{m}$, by essentially using a linear function of carrier energy to approximate the hyperbolic tangent function, ${ }^{[67]}$ which is especially inaccurate around the extreme points.

Tang et al. ${ }^{[67,68]}$ have found that the highest achievable thermopower $\left(S_{m}\right)$ of a materials system given an electronic band structure is determined by the sensitivity of transport rate to carrier energy, which is called the energy sensitivity of carrier transport $(s)$. Therefore, if we are using the constant relaxation time approximation or only considering the acoustic phonon scattering, we would be presuming the energy sensitivity to be fixed at 0 , which prohibits us to obtain the value of the highest achievable thermopower.

The density of free charge carriers passing across a unit area per unit of time is characterized by the transport rate $(\theta)$, and the energy sensitivity of carrier transport $(s)$ is represented as the relative change of transport rate per unit relative change of carrier energy $(\varepsilon)$ counting from the corresponding band edge $s=(d \theta / \theta) /(d \varepsilon / \varepsilon)$. The value of such an energy sensitivity is determined by the specific materials system and the temperature and is measured to be around $\sim 1.00$ for electrons and holes in graphene and carbon nanotubes at $300 \mathrm{~K}^{[67,69,70]}$ and around $\sim 0.50$ at $150 \mathrm{~K} .{ }^{[67,68]}$ The asymmetry ratio between the transport rates of holes and electrons is measured to be in the range of $1.50 \sim 2.00$ at $150 \sim 300 \mathrm{~K} .{ }^{[67,69,70]}$ The electrical conductivity and the thermopower can be generally calculated $\operatorname{as}^{[67]}$

$$
\sigma=e^{2} \sum_{\text {Bands }} \int q \theta\left(-\frac{\partial f}{\partial E}\right) d E
$$

and

$$
S=-\frac{k_{B}}{e^{2}} \frac{\sum_{\text {Bands }} \int q \theta\left(-\frac{\partial f}{\partial E}\right)\left(\frac{E-E_{f}}{k_{B} T}\right) d E}{\sum_{\text {Bands }} \int q \theta\left(-\frac{\partial f}{\partial E}\right) d E}
$$

where $k_{B}$ is the Boltzmann constant, $e$ is the elementary charge, $q$ of either $-e$ or $+e$ corresponds to the charge of each electron or hole, $f$ is the Fermi-Dirac distribution and $E_{f}$ is the Fermi level. Fortunately, this method of thermopower calculation is valid for the elastic and inelastic diffusive transport, ${ }^{[71-74]}$ the ballistic transport, ${ }^{[75-79]}$ and the quantum transport. ${ }^{[80-83]}$ Within each transport regime, the calculation of transport rate $(\theta)$ can be different. Commonly used methods include the Boltzmann equation, ${ }^{[84-87]}$ Rode's method, ${ }^{[88-93]}$ and the quantum transmission matrices. ${ }^{[94-98]}$ The active thermal conductivity is different than and imposed upon the passive thermal conductivity of $\kappa$ that is contributed by both lattice and charge carriers. Zebarjadi et al. ${ }^{[51]}$ have pointed out that though the energy efficiency of a thermoelectricity generator prefers an enhanced figure of merit, the solid-state thermoelectric refrigeration and cooling also prefers thermoelectric materials with large values of both thermal conductivity and/or power factor. For the application of refrigeration, the direction of active thermal conduction is opposite to natural heat flux, i.e. parallel to the temperature gradient, which can be as high as $^{[51,61]}$

$$
r_{a, r}=\frac{\sigma S^{2} T_{c}}{2 \Delta T}
$$

where $T_{C}$ and $T_{H}$ are the cold-side and hot-side temperatures, respectively, and $\Delta T=T_{H}-T_{C}$ is the overall temperature drop. The values of $\sigma$ and $S$ are corresponding to the mid-point temperature $T_{\text {mid }}=\left(T_{H}+T_{C}\right) / 2$. For the application of cooling, the direction of active thermal conduction is, instead, parallel to the natural heat flux, and the expression of its highest achievable value is slightly different as ${ }^{[51,61]}$

$$
r_{a, c}=\frac{\sigma S^{2} T_{H}}{2 \Delta T}
$$

The passive thermal conductivity and the active refrigeration/cooling rate for a single one-dimensional material such as a carbon nanotube can be measured by forming a nanoscale bridge structure according to previous reports. ${ }^{[28,99-101]}$

\section{Results and discussions}

We have studied the influence of band gap on the highest achievable active thermoelectric refrigeration and cooling rate of carbon nanotubes. To make the comparison as fair as possible, we have chosen three individual single-wall carbon nanotubes with similar diameters, but different electronic band gaps, whose chirality indices are $(5,5),(6,3)$, and $(8,0)$, respectively. ${ }^{[30,31,35-41]}$

The (5,5)-carbon nanotube has a diameter of $d=6.78 \AA$, and a zero-valued band-gap, which exhibits a metallic transport behavior. The electrical conductivity and the thermopower as a function of the Fermi level at the room temperature of $300 \mathrm{~K}$ are calculated based on Equations (1) and (2) and shown in Fig. 1(a). The electrical conductivity has its minimum value when the Fermi level is aligned with the boundary between the conduction band and the valence band, which is still as high as $1.33 \times 10^{7} \mathrm{~S} / \mathrm{m}$. The highest achievable values of thermopower are $1.03 \times 10^{-4} \mathrm{~V} / \mathrm{K}$ for the $p$-type region and $0.73 \times 10^{-4} \mathrm{~V} / \mathrm{K}$ for the $n$-type region, respectively. Such results are comparable to the values measured in metallic graphene samples at the same temperature of $0.93 \times 10^{-4} \mathrm{~V} / \mathrm{K}$ (p-type) and $0.60 \times 10^{-4} \mathrm{~V} / \mathrm{K}$ (n-type). ${ }^{[67,69]} \mathrm{Fig} .1(\mathrm{~b})$ 

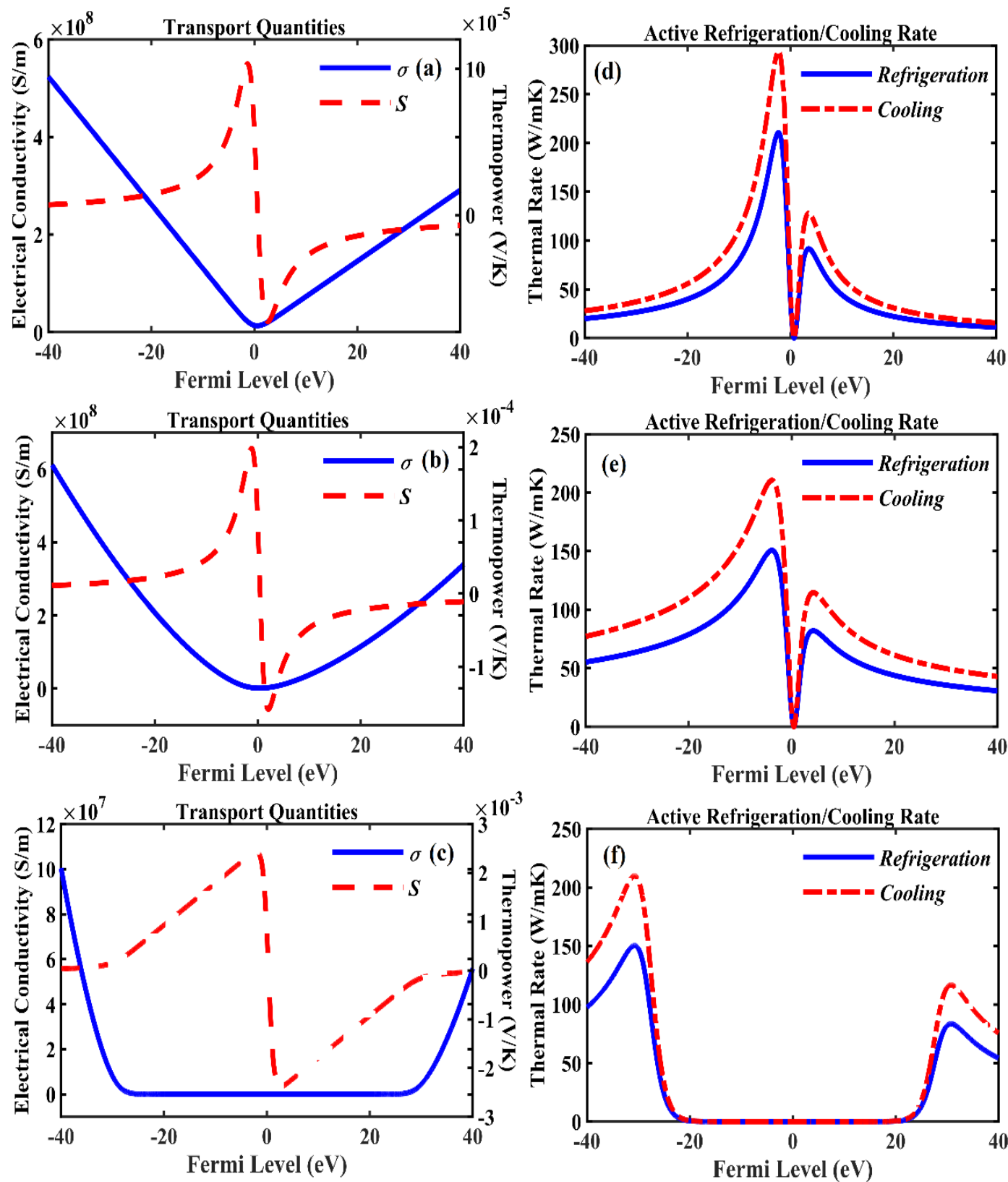

Fig. 1 The curves in (a), (b), and (c) are showing the calculated electrical conductivity and thermopower as a function of the Fermi level for the $(5,5)-,(6,3)$ - and $(8,0)$-carbon nanotubes, which are metallic, semi-metallic and semiconducting in transport, respectively. The results are more accurate compared to the traditional relaxation time approximation methods of diffusive transport, due to our incorporation of the bijection between the thermopower and the sensitivity of the transport rate to the carrier energy. In (e), (f), and (g), the rates of active Peltier refrigeration and cooling are calculated, corresponding to (a), (b), and (c), respectively.

corresponds to the $(6,3)$-carbon nanotube with a diameter of $d$ decreases from $1.33 \times 10^{7}$ to $1.49 \times 10^{6}$ and then to $2.59 \times 10^{6}$ $=6.22 \AA$, and a mini-band-gap of $0.057 \mathrm{eV}$, which is $\mathrm{S} / \mathrm{m}$, and the range of minimum conductivity widens up as the considered semi-metallic during the transport phenomenon at room temperature. Fig. 1(c) corresponds to the $(8,0)$-carbon nanotube with a diameter of $d=6.27 \AA$, and a considerable band-gap of $1.46 \mathrm{eV}$, which is obviously semiconducting. Such three single-wall carbon nanotubes have similar diameters in the order of $\sim 6 \AA$, so their differences in transport are considered to be resulting from the electronic band structure. The transition from metallic, to semi-metallic and then the semiconducting phase is apparent in the electrical conductivity, where the value of minimum conductivity band gap increases. The thermopower, on the other hand, increases with the band gap, which reaches $1.98 \times 10^{-4}$ for $p$ type and $1.58 \times 10^{-4} \mathrm{~V} / \mathrm{K}$ for $n$-type in the $(6,3)$-carbon nanotube and then $2.43 \times 10^{-3}$ for $p$-type and $2.38 \times 10^{-3} \mathrm{~V} / \mathrm{K}$ for $n$-type in the $(8,0)$-carbon nanotube, based on Equations (1) and (2). However, it is interesting to observe that the degree of asymmetry of the highest thermopower between the $p$-type and $n$-type regimes is decreasing with the band gap from 1.42 to 1.25 and then to 1.02 . The thermopower for the $(8,0)$ carbon nanotube is almost symmetric between the holes and electrons, 
despite its asymmetrical electrical conductivity.

We then investigated the active rates of thermoelectric refrigeration and cooling, respectively, for the three carbon nanotubes at the room temperature of $300 \mathrm{~K}$. To ensure a meaningful comparison, we assume all the refrigeration and cooling to be operating between $T_{H}=325 \mathrm{~K}$ (hot side) and $T_{C}$ $=275 \mathrm{~K}$ (cold side), and the temperature drop is, therefore, $\Delta T$ $=50 \mathrm{~K}$. The data are shown in Figs. 2(d), (e), and (f) correspond to the $(5,5),(6,3)$, and $(8,0)$-carbon nanotube, respectively, for both the $p$-type and the $n$-type cases. As shown in the figures, the active rate for cooing is slightly higher than the refrigeration, but generally not noticeably different in all of the three carbon nanotubes. The active rates are also comparable with the thermal conductivity of commonly used thermal conducting metals at the same temperature, e.g. copper $(386 \mathrm{~W} / \mathrm{mK})$, aluminum $(239 \mathrm{~W} / \mathrm{mK})$, and gold $(310 \mathrm{~W} / \mathrm{mK}),{ }^{[102]}$ which implies a good materials system for heat management. The performance is expected to be even better when the temperature drop is smaller, which will be discussed later. Although the electrical conductivity and the thermopower differ significantly in the three metallic, semi-metallic, and semiconducting carbon nanotubes, the maximum rates of active thermoelectric refrigeration and cooling almost all fall into the range of $200 \sim 300 \mathrm{~W} / \mathrm{mK}$. This information is helpful for the large-scale synthesis of such thermal management devices since the insensitivity of the active rates to the carbon nanotube band-gap is ensuring the robustness of performance with respect to the unavoidable variation of chirality/band-gap distribution in batch manufacturing.

During the design of thermoelectric refrigeration or cooling devices, it is preferred that the $p$-leg and the $n$-leg have as similar performance as possible. However, as in the cases of most Peltier refrigeration and cooling materials systems, the performance of the $p$-type regime is different from the $n$-type region in carbon nanotubes as well. The hole-electron asymmetry ratio between the highest achievable active refrigeration/cooling rates are $2.30,1.84$, and 1.24 for the $(5,5)$, $(6,3)$, and $(8,0)$-carbon nanotubes at room temperature. Such asymmetry ratios are, however, smaller than the values in other commonly used large- $Z T$ thermoelectric materials e.g. PbTe $(8.79)^{[103]}$ and $\mathrm{BaSnO}_{3}\left(5.32 \times 10^{3}\right),{ }^{[104]}$ which further implies a promising potential for the ultimate manufacturing of Peltier refrigeration and cooling systems based on carbon nanotubes.

Figs. 2 (a), (b), and (c) are illustrating our calculated results on how the temperature drop affects the best performance of active refrigeration and cooling, for the metallic, the semimetallic, and the semiconducting carbon nanotubes, respectively. It is seen that the smaller the temperature drop that the heat managing device is working over, the better the performance is expected to be. When the temperature drop is as small as $10 \mathrm{~K}$, the active refrigeration and cooling rate can possibly achieve as high as $\sim 2500 \mathrm{~W} / \mathrm{mK}$, which is $\sim 6.5$ times the thermal conductivity of copper. ${ }^{[102]}$ When the temperature drop is widened, the active rate is generally declining. However, the refrigeration or cooling systems in practice are usually cascaded into different small-range temperature windows to ensure overall performance. For all these temperature windows shown in Fig. 2, the metallic carbon nanotube behaves slightly better than the semi-metallic and then the semiconducting carbon nanotubes. However, the difference is still not significant, which again ensures the stability of performance at a variety of temperature windows, against the fluctuation of the band gap during the batch synthesis of carbon nanotubes.
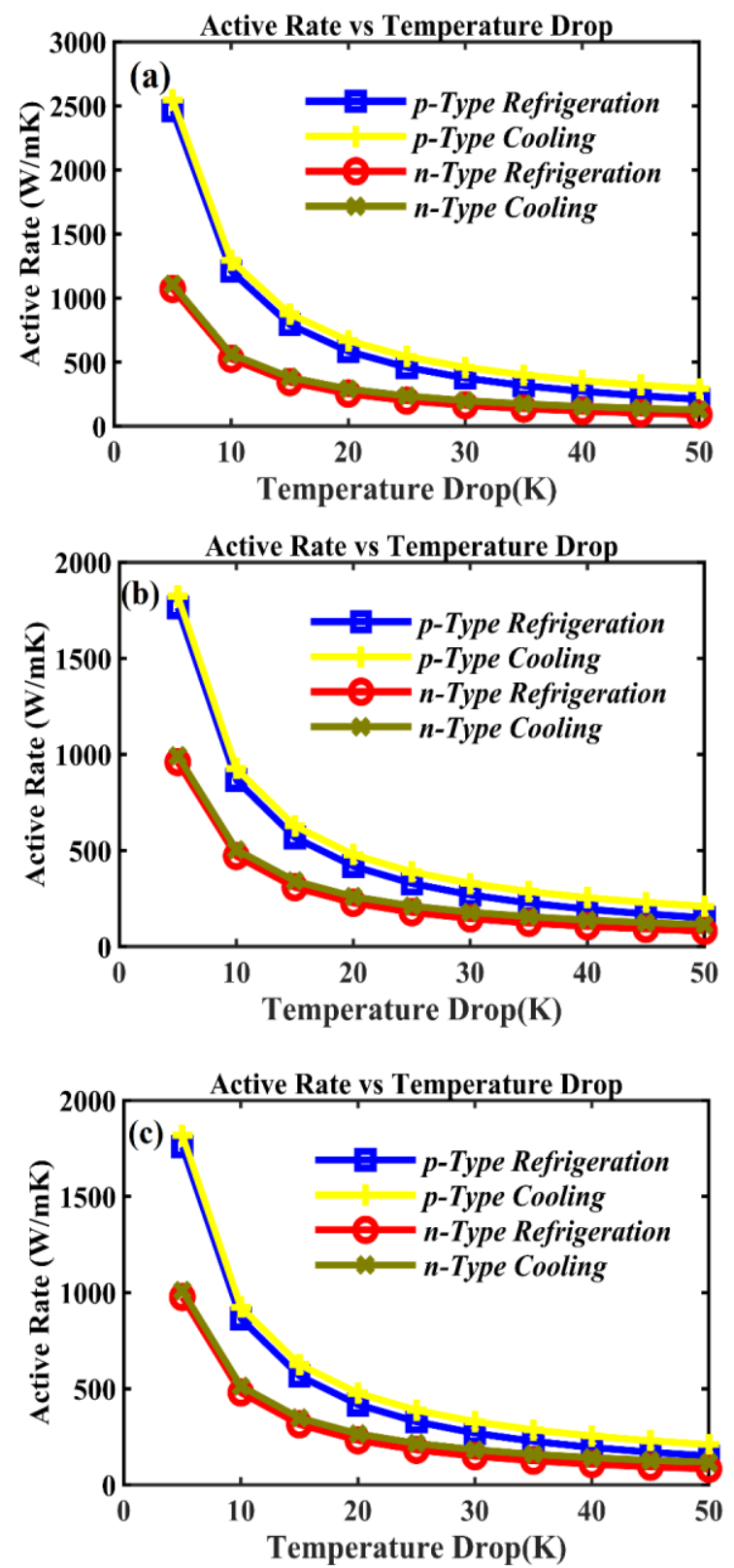

Fig. 2 The highest achievable rates of active solid-state refrigeration and cooling out of the Peltier effect for the $(5,5)$-, $(6,3)$ - and $(8,0)$-carbon nanotubes are calculated for both the $p$ type and the $n$-type regimes, in (a), (b) and (c), respectively. The mid-point temperature for all the data points is set at $T_{\text {mid }}=300$ $\mathrm{K}$. The temperature drop marks the difference between the hot side and the cold side, i.e. $\Delta T=T_{H^{-}} T_{C}$. 

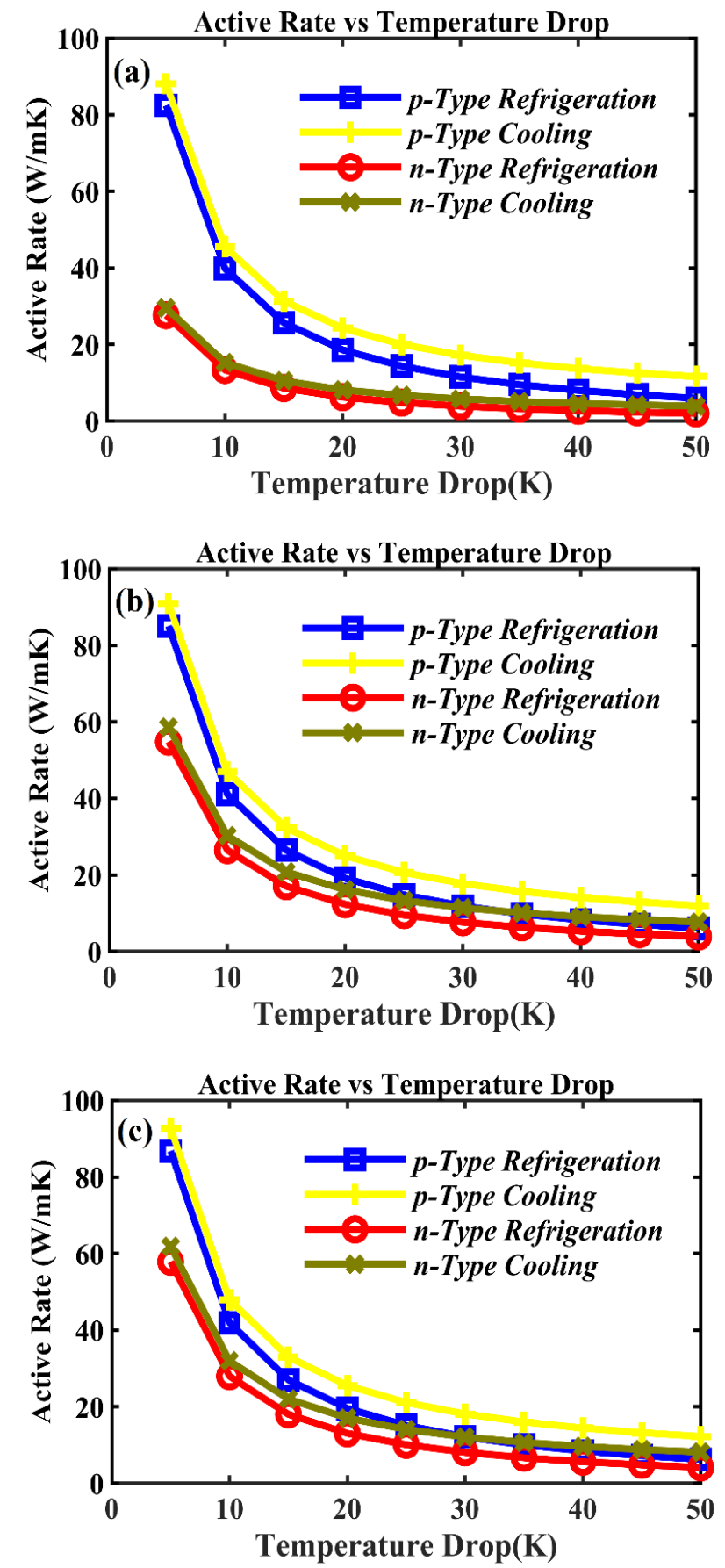

Fig. 3 Here we are considering the temperature influence on the refrigeration and cooling performance of carbon nanotubes. Compared to Fig 2, everything else is the same except that the mid-point temperature is now set to be $T_{\text {mid }}=150 \mathrm{~K}$.

Beyond the room temperature scenario, we have also studied the active rates for Peltier refrigeration and cooling at a cryogenic temperature of $150 \mathrm{~K}$, as shown in Fig. 3. The heat management at this temperature is expected to be more difficult, as can be seen from Fig. 3, the active rates are significantly smaller than the values at the room temperature shown in Fig. 2. However, the highest achievable refrigeration and cooling rates are still in the same order of magnitude $\left(\sim 10^{2}\right.$ $\mathrm{W} / \mathrm{mK})$ as many commonly used thermal conducting metals. When we compare the cases of the metallic, the semi-metallic, and semiconducting carbon nanotubes, we can see that the performance is still not sensitive to the variance of band-gap, which again is positive information for the industrialization of the device manufacturing.

\section{Conclusions}

In conclusion, we have studied the promising potential of carbon nanotubes in solid-state heat management for micro and mesoscale systems. The active rate for refrigeration and cooling at the room temperature of $300 \mathrm{~K}$ and the cryogenic temperature of $150 \mathrm{~K}$ is investigated. We have found that such active rates are as higher as, or better than, the commonly used thermal conducting metals. It is also interesting to see that the refrigeration and cooling performance are relatively robust against the fluctuation of the band gap in the batch producing of carbon nanotubes, which is useful for the design and possible industrialization of these small-scale systems. Furthermore, as the thermal conductivity of normal metals decreases with temperature, while the active refrigeration and cooling rate of carbon nanotubes are having an opposite trend, our suggested thermoelectric materials appear especially attractive for managing a heat dumping system at or above room temperature. The same methodology can also be used in other nanomaterials such as nanowires, nanoribbons, and nanorods, of SiGe, $\mathrm{MoS}_{2}$, and tellurium.

\section{Conflict of Interest}

There are no conflicts to declare.

\section{Supporting information}

Not applicable.

\section{References}

[1] J. Seidel, Nature Materials, 2019, 18, 188-190, doi: 10.1038/s41563-019-0301-z.

[2] Y. Y. Illarionov, T. Knobloch, M. Jech, M. Lanza, D. Akinwande, M. I. Vexler, T. Mueller, M. C. Lemme, G. Fiori, F. Schwierz, T. Grasser, Nature Communications, 2020, 11, 3385, doi: 10.1038/s41467-020-16640-8.

[3] A. H. Atabaki, S. Moazeni, F. Pavanello, H. Gevorgyan, J. Notaros, L. Alloatti, M. T. Wade, C. Sun, S. A. Kruger, H. Meng, K. Al Qubaisi, I. Wang, B. Zhang, A. Khilo, C. V. Baiocco, M. A. Popović, V. M. Stojanović, R. J. Ram, Nature, 2018, 556, 349354, doi: 10.1038/s41586-018-0028-z.

[4] Y. Liu, J. Zhang, L. M. Peng, Nature Electronics, 2018, 1, 644651, doi: 10.1038/s41928-018-0176-z.

[5] T. Tan, X. Jiang, C. Wang, B. Yao, H. Zhang, Advanced Science, 2020, 7, 2000058, doi: 10.1002/advs. 202000058.

[6] K. Y. Tan, M. Partanen, R. E. Lake, J. Govenius, S. Masuda, M. Möttönen, Nature Communications, 2017, 8, 15189, doi: 10.1038/ncomms 15189.

[7] C. Mukhopadhyay, A. Misra, S. Bhattacharya, A. K. Pati, Physical Review E, 2018, 97, 062116, doi: 10.1103/physreve.97.062116.

[8] Y. An, C. Sheng, X. Li, Nanoscale, 2019, 11, 17073-17083, 
doi: 10.1039/c9nr04110a.

[9] D. H. Nguyen, H. S. Ahn, International Journal of Heat and Mass Transfer, 2021, 178, 121601, doi: 10.1016/j.ijheatmasstransfer.2021.121601

[10] J. L. Smoyer, P. M. Norris, Heat Transfer Engineering, 2019, 40, 269-282, doi: 10.1080/01457632.2018.1426265.

[11] X. Zianni, Advanced Electronic Materials, 2021, 7, 2100176, doi: 10.1002/aelm.202100176.

[12] S. Bhanushali, N. N. Jason, P. Ghosh, A. Ganesh, G. P. Simon, W. Cheng, ACS Applied Materials \& Interfaces, 2017, 9, 18925 18935, doi: 10.1021/acsami.7b03339.

[13] Z. Barani, A. Mohammadzadeh, A. Geremew, C.-Y. Huang, D. Coleman, L. Mangolini, F. Kargar, A. A. Balandin, Advanced Functional Materials, 2020, 30, 1904008, doi: 10.1002/adfm.201904008.

[14] L. Lei, L. Bolzoni, F. Yang, Carbon, 2020, 168, 553-563, doi: 10.1016/j.carbon.2020.07.001.

[15] K. I. Uchida, S. Daimon, R. Iguchi, E. Saitoh, Nature, 2018, 558, 95-99, doi: 10.1038/s41586-018-0143-x.

[16] W. Jin, L. Liu, T. Yang, H. Shen, J. Zhu, W. Xu, S. Li, Q. Li, L. Chi, C. A. Di, D. Zhu, Nature Communications, 2018, 9, 3586, doi: 10.1038/s41467-018-05999-4.

[17] J. L. Blackburn, A. J. Ferguson, C. Cho, J. C. Grunlan, Advanced Materials, 2018, 30, 1704386, doi: 10.1002/adma.201704386.

[18] X. Hu, X. Gong, M. Zhang, H. Lu, Z. Xue, Y. Mei, P. K. Chu, Z. An, Z. Di, Small, 2020, 16, 1907170, doi: 10.1002/smll.201907170.

[19] M. Ikhlas, T. Tomita, T. Koretsune, M. T. Suzuki, D. NishioHamane, R. Arita, Y. Otani, S. Nakatsuji, Nature Physics, 2017, 13, 1085-1090, doi: 10.1038/nphys4181.

[20] A. Sakai, Y. P. Mizuta, A. A. Nugroho, R. Sihombing, T. Koretsune, M. T. Suzuki, N. Takemori, R. Ishii, D. NishioHamane, R. Arita, P. Goswami, S. Nakatsuji, Nature Physics, 2018, 14, 1119-1124, doi: 10.1038/s41567-018-0225-6.

[21] J. Xu, W. A. Phelan, C. L. Chien, Nano Letters, 2019, 19, 8250-8254, doi: 10.1021/acs.nanolett.9b03739.

[22] S. Tang, ES Materials \& Manufacturing, 2019, 4, 45-50, doi: 10.30919/esmm5f213.

[23] D. Beretta, N. Neophytou, J. M. Hodges, M. G. Kanatzidis, D. Narducci, M. M. Gonzalez, M. Beekman, B. Balke, G. Cerretti, W. Tremel, A. Zevalkink, A. I. Hofmann, C. Müller, B. Dörling, M. C. Quiles, M. Caironi, Materials Science and Engineering. Reports, 2019, 138, 100501, doi: 10.1016/j.mser.2018.09.001.

[24] Y. Xiao, L. D. Zhao, Science, 2020, 367, 1196-1197, doi: 10.1126/science.aaz9426.

[25] S. Y. Yue, T. Ouyang, M. Hu, Scientific reports, 2015, 5, 1 8, doi: $10.1038 /$ srep 14178

[26] B. Kumanek, D. Janas, Journal of Materials Science, 2019, 54, 7397-7427, doi: 10.1007/s10853-019-03368-0.

[27] D. Bruns, A. Nojeh, A. S. Phani, J. Rottler, Physical Review B, 2020, 101, 195408, doi: 10.1103/physrevb.101.195408.

[28] C. Yu, L. Shi, Z. Yao, D. Li, A. Majumdar, Nano Letters, 2005, 5, 1842-1846, doi: 10.1021/n1051044e.

[29] M. B. Jakubinek, M. A. White, G. Li, C. Jayasinghe, W. Cho,
M. J. Schulz, V. Shanov, Carbon, 2010, 48, 3947-3952, doi: 10.1016/j.carbon.2010.06.063.

[30] M. S. Dresselhaus, G. Dresselhaus, P. C. Eklund, A. M. Rao, Carbon nanotubes. Physics and Chemistry of Materials with LowDimensional Structures, 2000, 331-379, doi: 10.1007/978-94011-4038-6 9.

[31] F. Yang, M. Wang, D. Zhang, J. Yang, M. Zheng, Y. Li, Chemical Reviews, 2020, 120, 2693-2758, doi: 10.1021/acs.chemrev.9b00835.

[32] S. Kumar, M. Nehra, D. Kedia, N. Dilbaghi, K. Tankeshwar, K. H. Kim, Progress in Energy and Combustion Science, 2018, 64, 219-253, doi: 10.1016/j.pecs.2017.10.005.

[33] D. Janas, Materials Chemistry Frontiers, 2018, 2, 36-63, doi: 10.1039/c7qm00427c.

[34] N. Gupta, S. M. Gupta, S. K. Sharma, Carbon Letters, 2019, 29, 419-447, doi: 10.1007/s42823-019-00068-2.

[35] Z. Zhou, M. Steigerwald, M. Hybertsen, L. Brus, R. A. Friesner, Journal of the American Chemical Society, 2004, 126, 3597-3607, doi: 10.1021/ja039294p.

[36] X. Zheng, G. Chen, Z. Li, S. Deng, N. Xu, Physical Review Letters, 2004, 92, 106803, doi: 10.1103/physrevlett.92.106803.

[37] K. Kamaras, M. E. Itkis, H. Hu, B. Zhao, R. C. Haddon, Science, 2003, 301, 1501, doi: 10.1126/science.1088083.

[38] N. G. Lebedev, I. V. Zaporotskova, L. A. Chernozatonskii, International Journal of Quantum Chemistry, 2004, 100, 548-558, doi: 10.1002/qua.20111.

[39] D. C. Sorescu, K. D. Jordan, P. Avouris, The Journal of Physical Chemistry B, 2001, 105, 11227-11232, doi: 10.1021/jp0122979.

[40] P. Jarillo-Herrero, S. Sapmaz, C. Dekker, L. P. Kouwenhoven, H. S. J. van der Zant, Nature, 2004, 429, 389-392, doi: 10.1038 /nature 02568 .

[41] M. Steiner, M. Freitag, V. Perebeinos, A. Naumov, J. P. Small, A. A. Bol, P. Avouris, Nano Letters, 2009, 9, 3477-3481, doi: $10.1021 / \mathrm{n} 19016804$.

[42] J.-P. Salvetat-Delmotte, A. Rubio, Carbon, 2002, 40, 17291734, doi: 10.1016/s0008-6223(02)00012-x.

[43] K. Mylvaganam, L. C. Zhang, Carbon, 2004, 42, 2025-2032, doi: 10.1016/j.carbon.2004.04.004.

[44] X. Zhang, W. Lu, G. Zhou, Q. Li, Advanced Materials, 2020 , 32, 1902028, doi: 10.1002/adma.201902028.

[45] B. Wang, W. Huang, L. Chi, M. Al-Hashimi, T. J. Marks, A. Facchetti, Chemical Reviews, 2018, 118, 5690-5754, doi: 10.1021/acs.chemrev.8b00045.

[46] E. A. Scott, J. T. Gaskins, S. W. King, P. E. Hopkins, APL Materials, 2018, 6, 058302, doi: 10.1063/1.5021044.

[47] S. Zeng, Z. Tang, C. Liu, P. Zhou, Nano Research, 2021, 14, 1752-1767, doi: 10.1007/s12274-020-2945-Z.

[48] K. Yanagi, S. Kanda, Y. Oshima, Y. Kitamura, H. Kawai, T. Yamamoto, T. Takenobu, Y. Nakai, Y. Maniwa, Nano Letters, 2014, 14, 6437-6442, doi: 10.1021/nl502982f.

[49] H. Nishidome, K. Nagai, K. Uchida, Y. Ichinose, Y. Yomogida, Y. Miyata, K. Tanaka, K. Yanagi, Nano Letters, 2020, 20, 6215-6221, doi: 10.1021/acs.nanolett.0c02717.

[50] Y. Ichinose, M. Matsubara, Y. Yomogida, A. Yoshida, K. Ueji, 
K. Kanahashi, J. Pu, T. Takenobu, T. Yamamoto, K. Yanagi, Physical Review Materials, 2021, 5, 025404, doi: 10.1103/physrevmaterials.5.025404.

[51] M. Zebarjadi, Applied Physics Letters, 2015, 106, 203506, doi: 10.1063/1.4921457.

[52] E. M. Silverman, Northrop Grumman Technology Review Journal, 2005, 13, 1-19.

[53] L. Boukhris, A. Boudjemai, A. Bellar, R. Roubache, M. Bensaada, International Journal of Aerospace and Mechanical Engineering, 2011, 5, 2459-2461.

[54] C. Pan, Q. Youshan, L. Huijuan, T. Jiahai, in 2011 International Conference on Electronics, Communications and Control (ICECC)- (IEEE), 2011, 2615-2619.

[55] J. Cho, K. E. Goodson, Nature Materials, 2015, 14, 136-137, doi: $10.1038 /$ nmat4194.

[56] S. M. Sohel Murshed, C. A. Nieto de Castro, Renewable and Sustainable Energy Reviews, 2017, 78, 821-833, doi: 10.1016/j.rser.2017.04.112.

[57] M. Pino, J. J. García-Ripoll, Physical Review A, 2020, 101, 032324, doi: 10.1103/physreva.101.032324.

[58] S. Zaiser, C. T. Cheung, S. Yang, D. B. R. Dasari, S. Raeisi, J. Wrachtrup, Npj Quantum Information, 2021, 7, 92, doi: 10.1038/s41534-021-00408-z.

[59] Y. Wang, M. Um, J. Zhang, S. An, M. Lyu, J.-N. Zhang, L.M. Duan, D. Yum, K. Kim, Nature Photonics, 2017, 11, 646-650, doi: 10.1038/s41566-017-0007-1.

[60] S. Mobassem, N. J. Lambert, A. Rueda, J. M. Fink, G. Leuchs, H. G. L. Schwefel, Quantum Science and Technology, 2021, 6, 045005, doi: 10.1088/2058-9565/ac0f36.

[61] A. Ziabari, M. Zebarjadi, D. Vashaee, A. Shakouri, Reports on Progress in Physics, 2016, 79, 095901, doi: 10.1088/00344885/79/9/095901.

[62] M. Hirschberger, L. Spitz, T. Nomoto, T. Kurumaji, S. Gao, J. Masell, T. Nakajima, A. Kikkawa, Y. Yamasaki, H. Sagayama, H. Nakao, Y. Taguchi, R. Arita, T. H. Arima, Y. Tokura, Physical Review Letters, 2020, 125, 076602, doi: 10.1103/physrevlett.125.076602.

[63] H. Yamashita, K. Tsunoda, H. Nishino, S. Sato, Journal of Applied Physics, 2021, 129, 173101, doi: 10.1063/5.0036297.

[64] X. Jiang, C. Ban, L. Li, C. Wang, W. Chen, X. Liu, AIP Advances, 2021, 11, 055120, doi: 10.1063/5.0042555.

[65] M. A. B. Hamid, C. Kar Tim, Y. Bin Yaakob, M. A. B. Hazan, Materials Research Express, 2019, 6, 055803, doi: 10.1088/20531591/ab01ea.

[66] R. Saito, A. Nugraha, E. Hasdeo, N. Hung, W. Izumida, Single-Walled Carbon Nanotubes, 2019, 165-188.

[67] S. Tang, H. Wang, Y. Zhang, A. Li, H. Xie, X. Liu, L. Liu, T. Li, F. Huang, X. Xie, M. Jiang, Scientific Reports, 2013, 3, 2666, doi: $10.1038 /$ srep02666.

[68] D. Xu, S. Tang, X. Du, Q. Hao, Carbon, 2019, 144, 601-607, doi: 10.1016/j.carbon.2018.12.080.

[69] Y. M. Zuev, W. Chang, P. Kim, Physical Review Letters, 2009, 102, 096807, doi: 10.1103/physrevlett.102.096807.

[70] G Lim, K. D Kihm, H. G. Kim, W. Lee, W. Lee, K. R. Pyun, S. Cheon, P. Lee, J. Y. Min, S. H. Ko. Nanomaterials. 2018 22,
557., doi: 10.3390/nano8070557

[71] Q. Chen, G. A. Fiete, Physical Review B, 2016, 93, 155125, doi: 10.1103/physrevb.93.155125.

[72] B. Z. Rameshti, R. Asgari, Physical Review B, 2016, 94, 205401, doi: 10.1103/physrevb.94.205401.

[73] M. Zare, B. Z. Rameshti, F. G. Ghamsari, R. Asgari, Physical Review B, 2017, 95, 045422, doi: 10.1103/physrevb.95.045422.

[74] D. Kim, R. Du, S.-Y. Yu, Y. Yin, S. Dong, Q. Li, S. E. Mohney, X. Li, S. Tadigadapa, Nanotechnology, 2020, 31, 365703, doi: 10.1088/1361-6528/ab97d2.

[75] T. Ando, H. Matsumura, T. Nakanishi, Physica B: Condensed Matter, 2002, 323, 44-50, doi: 10.1016/s09214526(02)00964-x.

[76] H. J. Li, W. G. Lu, J. J. Li, X. D. Bai, C. Z. Gu, Physical Review Letters, 2005, 95, 086601, doi: 10.1103/physrevlett.95.086601.

[77] P. Poncharal, C. Berger, Y. Yi, Z. L. Wang, W. A. de Heer, The Journal of Physical Chemistry B, 2002, 106, 12104-12118, doi: $10.1021 / \mathrm{jp} 021271 \mathrm{u}$.

[78] Z. Wu, Y. Xing, W. Ren, Y. Wang, H. Guo, Carbon, 2019, 149, 364-369, doi: 10.1016/j.carbon.2019.04.062.

[79] Y. Zhu, C. Chen, S. Wu, R. Cheng, L. Cheng, W. L. Zhou, Journal of Applied Physics, 2020, 128, 064302, doi: 10.1063/5.0009406.

[80] P. Gehring, J. M. Thijssen, H. S. J. van der Zant, Nature Reviews Physics, 2019, 1, 381-396, doi: 10.1038/s42254-0190055-1.

[81] C. Maier, T. Brydges, P. Jurcevic, N. Trautmann, C. Hempel, B. P. Lanyon, P. Hauke, R. Blatt, C. F. Roos, Physical Review Letters, 2019, 122, 050501, doi: 10.1103/PhysRevLett.122.050501.

[82] H. Zhang, D. E. Liu, M. Wimmer, L. P. Kouwenhoven, Nature Communications, 2019, 10, 5128, doi: 10.1038/s41467019-13133-1.

[83] S. Wang, B. C. Lin, A. Q. Wang, D. P. Yu, Z. M. Liao, Advances in Physics: X, 2017, 2, 518-544, doi: 10.1080/23746149.2017.1327329.

[84] M. A. Cabero, C. Guo, C. Wan, J. Hu, S. Liu, M. Zhao, L. Zhang, Q. Song, Q. Song, H. Wang, S. Tu, N. Li, L. Sheng, J. Chen, Y. Liu, B. Wei, J. Zhang, X. Han, H. Yu, D. Yu, The Journal of Physical Chemistry C, 2021, 125, 13167-13175, doi: 10.1021/acs.jpcc.1c02717.

[85] Y. Ge, R. Liu, Z. Shuai, Applied Physics Letters, 2021, 118, 123301, doi: 10.1063/5.0043863.

[86] H. Li, K. Hayashi, Y. Miyazaki, Scripta Materialia, 2018, 150, 130-133, doi: 10.1016/j.scriptamat.2018.03.018.

[87] J. Li, Y. Wang, G. Zhang, D. Chen, F. Ren, Journal of Applied Physics, 2019, 125, 035107, doi: 10.1063/1.5053710.

[88] D. Rode, Electron Mobility in Direct-Gap Polar Semiconductors, Physical Review B, 1970, 2, 1012, doi: 10.1103/PhysRevB.2.1012.

[89] N. Miller, E. E. Haller, G. Koblmüller, C. Gallinat, J. S. Speck, W. J. Schaff, M. E. Hawkridge, K. M. Yu, J. W. Ager, Physical Review B, 2011, 84, 075315, doi: 10.1103/physrevb.84.075315. 
[90] G. Ng, D. Vasileska, D. K. Schroder, Journal of Applied and institutional affiliations. Physics, 2009, 106, 053719, doi: 10.1063/1.3212532.

[91] A. T. Ramu, L. E. Cassels, N. H. Hackman, H. Lu, J. M. O. Zide, J. E. Bowers, Journal of Applied Physics, 2010, 107, 083707, doi: 10.1063/1.3366712.

[92] A. T. Ramu, J. E. Bowers, Applied Physics Letters, 2012, 101, 173905, doi: 10.1063/1.4764517.

[93] A. Faghaninia, J. W. Ager, C. S. Lo, Physical Review B, 2015, 91, 235123, doi: 10.1103/physrevb.91.235123.

[94] D. Vashaee, Y. Zhang, A. Shakouri, G. Zeng, Y. J. Chiu, Physical Review B, 2006, 74, 195315, doi: 10.1103/physrevb.74.195315.

[95] N. A. Zimbovskaya, Journal of Physics: Condensed Matter, 2016, 28, 183002, doi: 10.1088/0953-8984/28/18/183002.

[96] B. L. Li, K. Q. Chen, Carbon, 2017, 119, 548-554, doi: 10.1016/j.carbon.2017.04.069.

[97] Q. H. Al-Galiby, H. Sadeghi, D. Z. Manrique, C. J. Lambert, Nanoscale, 2017, 9, 4819-4825, doi: 10.1039/c7nr00571g.

[98] E. J. Guzmán, S. Molina-Valdovinos, O. Oubram, I. Rodríguez-Vargas, Journal of Applied Physics, 2020, 128, 224302, doi: 10.1063/5.0030209.

[99] P. Kim, L. Shi, A. Majumdar, P. L. McEuen, Physical Review Letters, 2001, 87, 215502, doi: 10.1103/physrevlett.87.215502.

[100] M. Fujii, X. Zhang, H. Xie, H. Ago, K. Takahashi, T. Ikuta, H. Abe, T. Shimizu, Physical Review Letters, 2005, 95, 065502, doi: 10.1103/physrevlett.95.065502.

[101] J. Moon, K. Weaver, B. Feng, H. Gi Chae, S. Kumar, J.-B. Baek, G. P. Peterson, Review of Scientific Instruments, 2012, 83, 016103, doi: 10.1063/1.3676650.

[102] R. B. Bird, Applied Mechanics Reviews, 2002, 55, R1-R4, doi: 10.1115/1.1424298.

[103] L. Xu, Y. Zheng, J. C. Zheng, Physical Review B, 2010, 82, 195102, doi: 10.1103/physrevb.82.195102.

[104] J. Li, Z. Ma, R. Sa, K. Wu, RSC Advances, 2017, 7, $32703-$ 32709, doi: 10.1039/c7ra05193j.

\section{Author information}

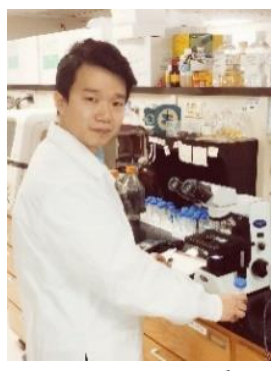

Dr. Shuang Tang joined the State University of New York Polytechnic Institute as a faculty member in 2015, after he received his $P h D$ in Materials Science and Engineering from the Massachusetts Institute of Technology, advised by Institute Professor Mildred Dresselhaus. His research interests include developing new materials systems based on nanoscience and nanotechnology for the application of sustainable energy, heat management, information technology, computer chips and satellite parts, and developing modern methods for doctoral, graduate and undergraduate education of science and engineering.

Publisher's Note: Engineered Science Publisher remains neutral with regard to jurisdictional claims in published maps 